CERN-TH/2001-082

hep-ph/0103248

\title{
The Cosmological Moduli Problem and Preheating
}

\author{
G.F. Giudice ${ }^{1}$, A. Riotto ${ }^{2}$, and I.I. Tkachev ${ }^{1}$ \\ ${ }^{1}$ CERN Theoretical Physics Division, \\ CH-1211 Geneva 23, Switzerland \\ ${ }^{2}$ INFN, Sezione di Padova, \\ Via Marzolo 8, Padova I-35131, Italy
}

\begin{abstract}
Many models of supersymmetry breaking, in the context of either supergravity or superstring theories, predict the presence of particles with Plancksuppressed couplings and masses around the weak scale. These particles are generically called moduli. The excessive production of moduli in the early Universe jeopardizes the successful predictions of nucleosynthesis. In this paper we show that the efficient generation of these dangerous relics is an unescapable consequence of a wide variety of inflationary models which have a preheating stage. Moduli are generated as coherent states in a novel way which differs from the usual production mechanism during parametric resonance. The corresponding limits on the reheating temperature are often very tight and more severe than the bound of $10^{9} \mathrm{GeV}$ coming from the production of moduli via thermal scatterings during reheating.
\end{abstract}

March 2001 


\section{Introduction}

In supergravity and (super)string models there exists a plethora of scalar and fermionic fields (we will loosely call them moduli $\Phi$ ) with masses of the order of the weak scale and gravitational-strength couplings to ordinary matter. If produced in the early Universe, such quanta behave like nonrelativistic matter and decay at very late times, eventually dominating the energy of the Universe until it is too late for nucleosynthesis to occur. This poses a serious cosmological problem [1]. Typical examples of these dangerous relics are the spin-3/2 gravitino (the supersymmetric partner of the graviton), the scalar moduli which parametrize supersymmetric flat directions in moduli space and seem almost ubiquitous in string theory, and their fermionic superpartners.

The slow decay rate of the $\Phi$-particles is the essential source of cosmological problems because the decay products of these relics will destroy the ${ }^{4} \mathrm{He}$ and $\mathrm{D}$ nuclei by photodissociation, and thus successful nucleosynthesis predictions [2, 3]. The most stringent bound comes from the resulting overproduction of $\mathrm{D}+{ }^{3} \mathrm{He}$; this requires that the $\Phi$-abundance relative to the entropy density $s$ at the time of reheating after inflation [4] should satisfy [5]

$$
\frac{n_{\Phi}}{s} \lesssim 10^{-12}
$$

where the exact bound depends upon the mass $m_{\Phi}$.

The dangerous moduli can be produced in the early Universe in a number of ways They may be generated by thermal scatterings in the plasma during the process of reheating after inflation when the energy density of the Universe gets converted into radiation. The number density $n_{\Phi}$ at thermalization is estimated to be of the order of $n_{\Phi} / s \simeq 10^{-2}\left(T_{r} / M_{\mathrm{Pl}}\right)$, where $T_{r}$ is the final reheating temperature and $M_{\mathrm{Pl}}=$ $1.2 \times 10^{19} \mathrm{GeV}$ is the Planck mass. This gives a stringent upper bound on the reheating temperature after inflation of about $10^{9} \mathrm{GeV}$ [3].

The generation of the dangerous relics may also proceed through non-thermal effects. Scalar moduli fields may be copiously created by the classical gravitational effects on the vacuum state between the end of inflation and the beginning of the matter/radiation phase [6, 7, 8]. In such a case, the upper bound on the reheating temperature is very dependent on the inflationary model, but it may be as low as $100 \mathrm{GeV}$. Another possibility is represented by the parametric excitation of dangerous relics occuring after inflation because of the rapid oscillations of the inflaton field(s). Gravitino production is an interesting example of this kind [7, 9, 10, 11] since the non-thermal generation of

\footnotetext{
${ }^{1}$ Here we will study only production of moduli quanta and we do not consider the possibility of moduli oscillations around their minimum.
} 
gravitinos in the early Universe can be extremely efficient and overcome the thermal production by several orders of magnitude?.

The scope of this paper is to show that the abundant production of dangerous relics is an unavoidable consequence of a period of preheating [13 after inflation. The corresponding limits on the reheating temperature are often very tight and more severe than the bound of $10^{9} \mathrm{GeV}$ coming from the production of moduli via thermal scatterings during reheating.

During the first stage of preheating nonperturbative quantum effects lead to an extremely effective dissipational dynamics and explosive particle production. Particles coupled to the inflaton field can be created in a broad parametric resonance with a fraction of the energy stored in the form of coherent inflaton oscillations at the end of inflation released after a few oscillations. This rapid transfer of energy density populates the Universe with quanta in highly nonthermal states which can be viewed either as classical waves travelling through the Universe [14] or as quantum particles in states with large occupation numbers. In the second stage of preheating, called semiclassical thermalization [14, rescatterings of the produced fluctuations smear out the resonance peaks in the power spectra and lead to a slowly evolving state in which the power spectra are smooth [14, 15, 16]. The system begins to exibit a chaotic behaviour characteristic of a classical non-linear system with many degrees of freedom. In the course of the subsequent slow evolution, the power spectra propagate to larger momenta, eventually leading to a fully thermalized state.

In supersymmetric and superstring theories coupling constants and masses appearing in the Lagrangian have to be thought as functions of the dimensionless ratio $\Phi / M_{\mathrm{Pl}}$. For instance, a generic coupling constant $h$ is in fact a function of the scalar moduli

$$
h(\Phi)=h\left(\frac{\langle\Phi\rangle}{M_{\mathrm{Pl}}}\right)\left(1+c \frac{\delta \Phi}{M_{\mathrm{Pl}}}+\cdots\right),
$$

where $c$ is a coefficient usually of order unity and $\delta \Phi=\Phi-\langle\Phi\rangle$. This expansion introduces a gravitational coupling between the scalar moduli and the matter fields. If the underlying theory is supersymmetric, similar couplings of the scalar field $\Phi$ and

\footnotetext{
${ }^{2}$ Recently, this statement has been criticized in ref. [12], where it was shown that, in a simple Polonyi model, gravitino production is suppressed. Undoubtedly, as emphasized in refs. [7, 9, 10, 11], the nonthermal gravitino production depends sensitively on the assumptions on the inflationary model and its couplings to the supersymmetry-breaking sector. Non-thermal production of gravitinos is expected to be significant and to pose a cosmological problem if - after inflation - there is a strong mixing between the inflaton sector and the supersymmetry-breaking sector (of the present vacuum). As it will become clear in the present paper, this is often the case because, during the first few inflaton oscillations when the value of the inflaton field is close to $M_{\mathrm{Pl}}$, generic Planck mass-suppressed interactions can give a large contribution to such mixing.
} 
its fermionic superpartner to the matter fields will arise in the superpotential or in the Kähler potential.

Since moduli fields couple to any form of matter at least gravitationally, the production is present in a wide variety of inflationary models which have a preheating stage. In general, the equation of motion for the (quantum) field $\delta \Phi$ during preheating is

$$
\square \delta \Phi+m^{2} \delta \Phi=\sum_{i} h_{i} J_{i}
$$

where the currents $J_{i}$ are functions of the (classical) fields created at preheating and have interaction vertices parametrized by the coupling constants $h_{i}$. In a cosmological setting particle creation in a time-varying classical background is usually caused by the time dependence of the effective mass of the field (consider, for instance, parametric resonance or gravitational creation of particles). As a result, particles are created in a squeezed state. On the contrary, creation of moduli is caused by the non-zero classical current and therefore they are created as coherent states. From a quantum mechanical point of view, this production mechanism is novel and differs from the more traditionally studied generation mechanism of squeezed states. Furthermore, the moduli production does not depend upon the particular properties of the modulus unlike the gravitino production [7, 9, 10, 11] and it is true both for scalar and fermionic moduli.

In this paper we perform a calculation of moduli production during the preheating stage in various inflationary models. In sect. 2 we consider the cases of chaotic inflation with quartic and quadratic inflaton potentials, with and without couplings of the inflaton to a new scalar field $X$. The case of hybrid inflation is discussed in sect. 3 , while sect. 4 contains a study of the production at preheating of modulinos, light fermions with only gravitational-strength couplings. In sect. 5 we summarize our results.

\section{Moduli Production at Preheating in Chaotic In- flation}

Let us first consider a simple model of chaotic inflation with quartic potential $V(\phi)=$ $\frac{\lambda}{4} \phi^{4}$. As discussed in sect. 1, we expect a generic coupling of the scalar moduli quanta $\delta \Phi$ with the inflaton field $\phi$ of the form $\lambda J \delta \Phi$, where $J \equiv c \phi^{4} /\left(4 M_{\mathrm{Pl}}\right)$. Let us denote the amplitude of the oscillating inflaton field as $\phi_{0}$, while the frequency of the oscillations is $\sim \sqrt{\lambda} \phi_{0}$, where $\phi_{0}$ is the zero-momentum mode of $\phi$.

In this model fluctuations of the inflaton field do not grow during chaotization (they actually decrease due to the redshift) so the most efficient production of moduli fields take place at the end of the resonance phase. As we noted, one can describe the states 
we are considering either as a collection of interacting classical waves or as a collection of particles in modes with large occupation numbers. Therefore at late times it is possible to treat the system classically and to solve the relevant equations of motion on a lattice. The initial conditions for the classical problem are found solving the preceding quantum evolution by means of the appropriate Bogolyubov transformations (for more details see Ref. [14]).

The results of the numerical integration on a $128^{3}$ lattice for $\lambda=10^{-12}$ are summarized in Fig. 1, where we plot the values of $\left\langle\phi^{2}\right\rangle,\left\langle\Phi^{2}\right\rangle$ and of the inflaton zero mode squared $\phi_{0}^{2}$ as a function of the conformal time [14] and in units $M_{\mathrm{Pl}}^{2}$. The field variance is given by $\left\langle\phi^{2}\right\rangle=\left[(2 \pi)^{3} \mathcal{V}\right]^{-1} \int d^{3} \mathbf{k} \phi_{\mathbf{k}}^{2}$, where $\mathcal{V}$ is the volume and $\phi_{\mathbf{k}}$ is the Fourier $\mathbf{k}$-mode of the field $\phi$. Here and in the following we have taken $c=1$. Time and particle momenta are given in units of $\sqrt{\lambda} \phi_{0}(0)$, where $\phi_{0}(0)$ is the initial value of the zero inflaton mode at the end of inflation. The time $t=0$, when inflation ends, is chosen as the moment of the first extremum of $\phi(t)$. The maximum value of $\left\langle\phi^{2}\right\rangle$ is achieved at the time $t_{r}$, which marks the end of the resonance phase, and, as shown in Fig. 1 is about $10^{-7} M_{\mathrm{Pl}}^{2}$, corresponding to $\left\langle\Phi^{2}\right\rangle \sim 10^{-15} M_{\mathrm{Pl}}^{2}$. At the same time the zero mode is about $\phi_{0}\left(t_{r}\right) \sim 10^{-3} M_{\mathrm{Pl}}$.

We will now present some analytical estimates which approximately reproduce the numerical results of Fig. 1. To obtain the intensity of the moduli gravitational production which accompanies the creation and annihilation of the fluctuations generated during preheating, we first express the solution of the modulus equation of motion in terms of the retarded Green function

$$
\Phi(x)=\Phi_{i n}(x)+\lambda \int d^{4} x^{\prime} G_{r e t}\left(x-x^{\prime}\right) J\left(x^{\prime}\right) .
$$

Here $\Phi_{i n}$ is the initial configuration at time $t=0$ and the retarded Green function is given by

$$
G_{r e t}\left(x-x^{\prime}\right)=\theta\left(t-t^{\prime}\right) \int \frac{d^{3} \mathbf{k}}{(2 \pi)^{3}} \frac{\sin \omega_{k}\left(t-t^{\prime}\right)}{\omega_{k}} e^{i \overrightarrow{\mathbf{k}} \cdot \overrightarrow{\mathbf{x}}} .
$$

In the case under consideration, the source term is given by $J=c \phi^{4} /\left(4 M_{\mathrm{Pl}}\right)$. Expanding the fields in Fourier modes, performing the integral over spatial coordinates, and taking $\phi_{0} \gg \delta \phi_{\mathbf{k}}$ (justified by the numerical results of Fig. 1), we obtain

$$
\Phi_{\mathbf{k}}(t)=\frac{c \lambda}{M_{\mathrm{Pl}}} \int_{0}^{t} d t^{\prime} \frac{\sin \left[\omega_{k}\left(t-t^{\prime}\right)\right]}{\omega_{k}} \phi_{0}^{3}\left(t^{\prime}\right) \delta \phi_{\mathbf{k}}\left(t^{\prime}\right),
$$

A crucial point is that the effect is linear in the inflaton fluctuations $\delta \phi_{\mathbf{k}}$. Therefore, the phenomenon of moduli production is due to the oscillations (similar to oscillations of neutrino species in a medium) of the inflaton quanta into moduli in presence of 


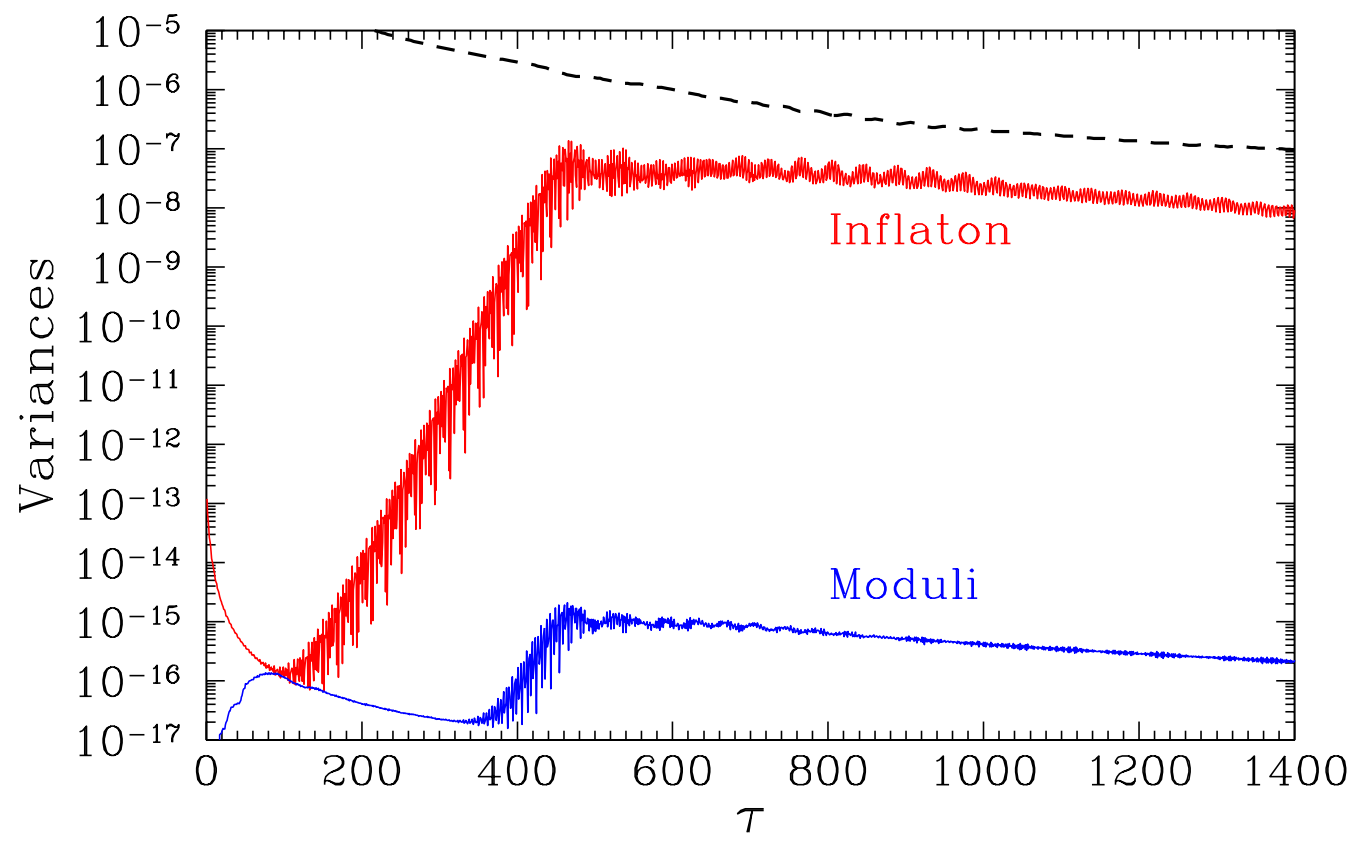

Figure 1: The variances of the inflaton and the moduli fields in units of $M_{\mathrm{Pl}}^{2}$ in a model with inflaton potential $V=\frac{\lambda}{4} \phi^{4}$ and moduli interaction $c \lambda \phi^{4} \delta \Phi /\left(4 M_{\mathrm{Pl}}\right)$, with $\lambda=10^{-12}$ and $c=1$. The dashed line shows the amplitude square of the inflaton field oscillations in the same units. 
the inflaton background $\phi_{0}$, rather than to real rescatterings. From a pure quantum mechanical point of view, scalar moduli are generated as coherent states and not as squeezed states as in the usual parametric resonance.

The fluctuations $\delta \phi_{\mathbf{k}}$ grow exponentially during the resonance phase as $e^{\mu t}$ where $2 \mu \simeq 0.07 \sqrt{\lambda} \phi_{0}(0)$ [14 and $\phi_{0}(0) \simeq 0.3 M_{\mathrm{Pl}}$ is the initial value of the inflaton field at the end of inflation. Therefore the integral in eq. (6) can be approximately evaluated by assuming that the exponential determines the full time dependence of the integrand. Then we obtain that the maximum value of the variance of the modulus field, achieved at the end of the resonance phase, is given by

$$
\left\langle\Phi^{2}\right\rangle \simeq \frac{c^{2} \lambda^{2} \phi_{0}^{6}\left(t_{r}\right)\left\langle\phi^{2}\right\rangle}{\omega_{k}^{2}\left(t_{r}\right) \mu^{2} M_{\mathrm{Pl}}^{2}} \simeq 10^{4} c^{2} \frac{\phi_{0}^{4}\left(t_{r}\right)}{M_{\mathrm{Pl}}^{4}}\left\langle\phi^{2}\right\rangle .
$$

Here we have used $\omega_{k}\left(t_{r}\right) \sim \sqrt{\lambda} \phi_{0}\left(t_{r}\right)$ as the typical frequency at the end of the resonance phase. Taking from Fig. 1 the values $\phi_{0} \sim 10^{-3} M_{\mathrm{Pl}}$ and $\left\langle\phi^{2}\right\rangle \sim 10^{-7} M_{\mathrm{Pl}}^{2}$ at $t=t_{r}$, eq. (7) gives $\left\langle\Phi^{2}\right\rangle \sim c^{2} 10^{-15} M_{\mathrm{Pl}}^{2}$, in very good agreement with the numerical result. Notice that the dependence on $\lambda$ dropped out from eq. (7). Our numerical calculation confirms that the maximum of $\left\langle\Phi^{2}\right\rangle$ is independent of the coupling $\lambda$ and it scales quadratically with c.

Since it is a good approximation to assume that the dominant particle production occurs around the time $t_{r}$, we find

$$
n_{\Phi} \sim \omega_{k}\left(t_{r}\right)\left\langle\Phi^{2}\right\rangle \text { and } n_{\phi} \sim \omega_{k}\left(t_{r}\right)\left\langle\phi^{2}\right\rangle .
$$

Using eq. (7) we obtain

$$
\frac{n_{\Phi}}{n_{\phi}} \sim 10^{-8} c^{2}\left[\frac{\phi_{0}\left(t_{r}\right)}{10^{-3} M_{\mathrm{Pl}}}\right]^{4}
$$

at the end of the resonance phase.

The ratio of the number density of moduli in units of the entropy density $s \sim \rho^{3 / 4} \sim$ $\lambda^{3 / 4} \phi_{0}^{3}\left(t_{r}\right)$ is given by

$$
\frac{n_{\Phi}}{s} \sim 10^{-6} c^{2}\left(\frac{10^{-13}}{\lambda}\right)^{1 / 4}\left(\frac{\phi_{0}^{2}\left(t_{r}\right)}{10^{-6} M_{\mathrm{Pl}}^{2}}\right)\left(\frac{\left\langle\phi^{2}\right\rangle}{10^{-7} M_{\mathrm{Pl}}^{2}}\right) .
$$

For $\lambda=10^{-13}$ (as fixed by the COBE normalization), this results contradicts the bound (11) by about six orders of magnitude (for $c$ of order unity) when the energy density in the scalar field is transferred to the energy density of a hot gas of relativistic particles. Notice that the ratio $\frac{n_{\Phi}}{s}$ does not depend on the time of thermalization, because both $n_{\Phi}$ and $V^{3 / 4}$ vary with the scale factor $a$ as $a^{-3}$.

In this calculation (and throughout all this paper) we have made the implicit hypothesis that the moduli fields are light during the preheating stage. However, the 


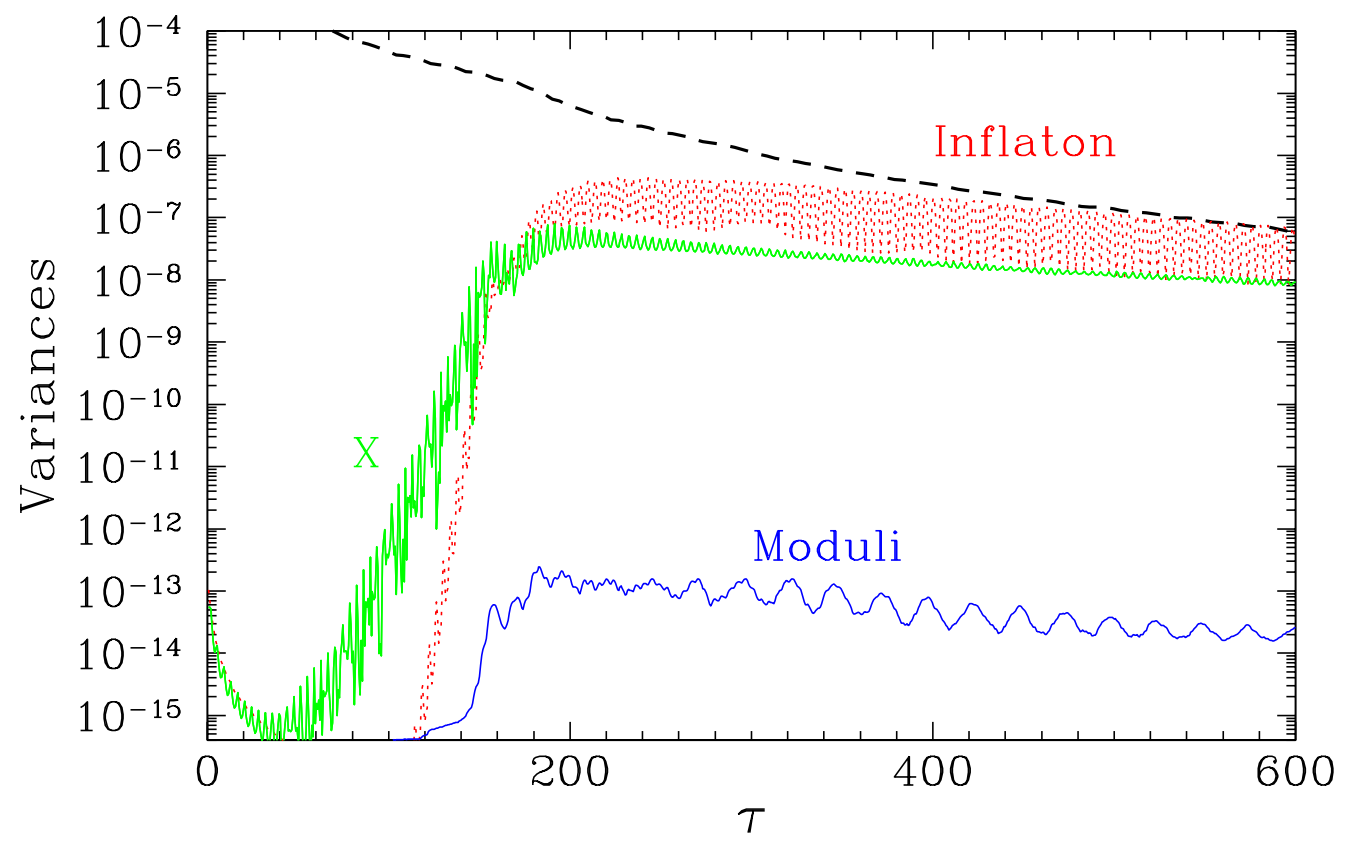

Figure 2: The variances of the inflaton, $X$ and moduli fields in units of $M_{\mathrm{Pl}}^{2}$ in a model with potential $V=\frac{\lambda}{4} \phi^{4}+\frac{g^{2}}{2} \phi^{2} X^{2}$ and moduli interaction $c \lambda \phi^{4} \delta \Phi /\left(4 M_{\mathrm{Pl}}\right)$, with $\lambda=10^{-12}, q \equiv g^{2} /(4 \lambda)=25$ and $c=1$. The dashed line shows the amplitude square of the inflaton field oscillations in the same units.

same generic expansion in eq. (2) is expected to provide the moduli fields with a mass of the order of the Hubble rate $H$ during the preheating stage. Consider the simple model of chaotic inflation with potential $V(\phi)=\frac{\lambda}{4} \phi^{4}$. The expansion of the coupling $\lambda\left(\Phi / M_{\mathrm{Pl}}\right)$ at the quadratic order in $\Phi / M_{\mathrm{Pl}}$ leads to a term in the Lagrangian of the form $\sim \lambda\left(\delta \Phi / M_{\mathrm{Pl}}\right)^{2} \phi^{4} \propto H^{2}(\delta \Phi)^{2}$. Nevertheless, our numerical and analytical estimates are not affected by treating the moduli as light states. This is because moduli fields are generically produced with frequencies $\omega$ much larger than the Hubble rate $\left(\omega \sim \sqrt{\lambda} \phi_{0}\left(t_{r}\right) \gg H\left(t_{r}\right)\right.$ in the chaotic model $\left.\lambda \phi^{4}\right)$ and kinematically they may be regarded as massless. This does not come as a surprise since it is known that superheavy particles may be easily generated at preheating [17].

A simple variation of the model considered so far is the one in which a massless inflaton field is coupled to another field $X, V=\frac{1}{4} \lambda \phi^{4}+\frac{1}{2} g^{2} \phi^{2} X^{2}$. The oscillating field $\phi$ amplifies fluctuations of the field $X$ via parametric resonance. The strength of the resonance can be parametrized by the $q$-parameter $q \equiv g^{2} / 4 \lambda$. Fluctuations of the inflaton field grow rapidly because of the back-reaction and the energy of the inflaton zero mode is quickly released into quanta of the $X$-field and the inflaton field. 


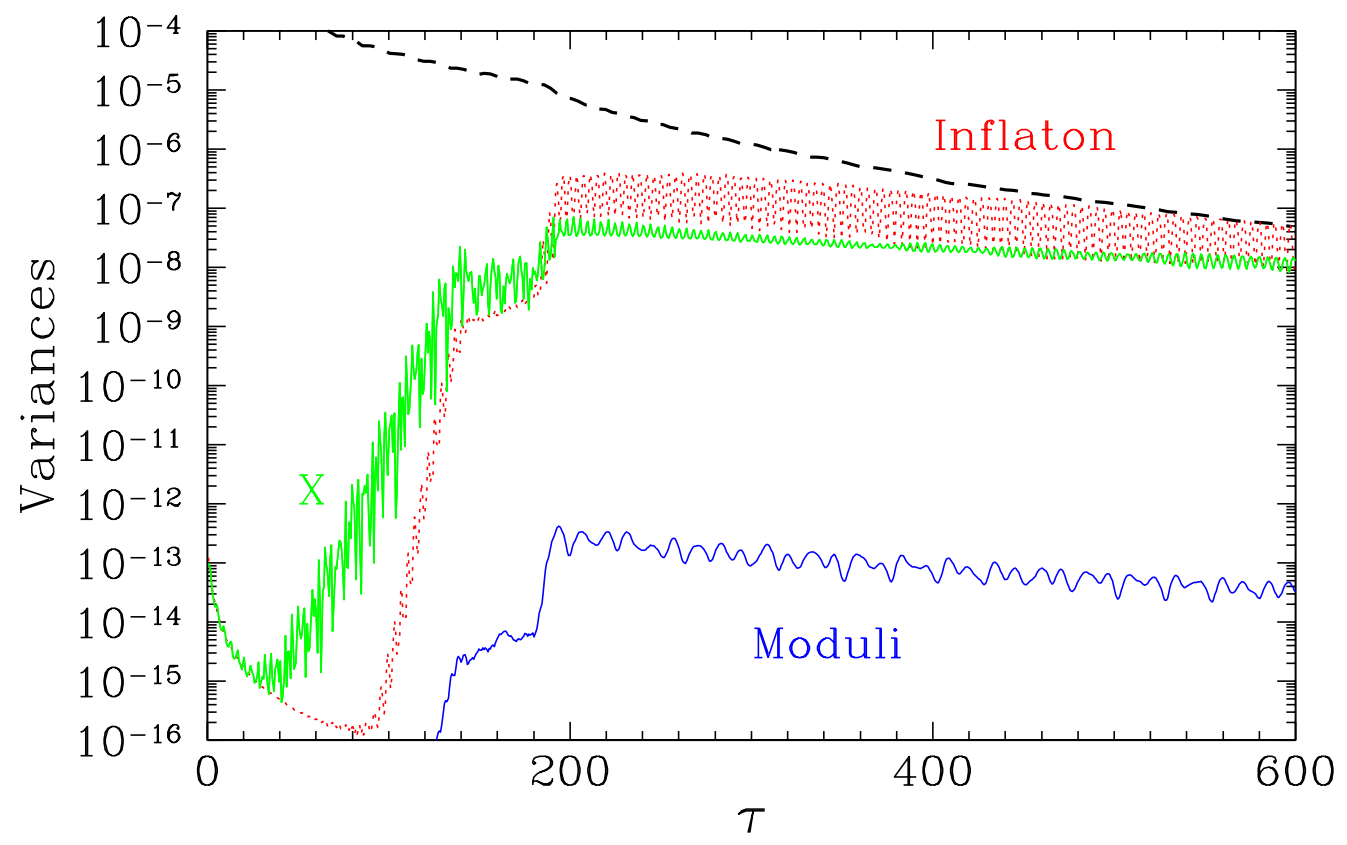

Figure 3: The variances of the inflaton, $X$ and moduli fields in units of $M_{\mathrm{Pl}}^{2}$ in a model with potential $V=\frac{\lambda}{4} \phi^{4}+\frac{g^{2}}{2} \phi^{2} X^{2}$ and moduli interaction $g^{2} \phi^{2} X^{2} \delta \Phi /\left(2 M_{\mathrm{Pl}}\right)$, with $\lambda=10^{-12}, q \equiv g^{2} /(4 \lambda)=25$. The dashed line shows the amplitude square of the inflaton field oscillations in the same units.

For $q=25$, we numerically find that the maximum value of $\left\langle\phi^{2}\right\rangle$ is about $5 \times 10^{-7} M_{\mathrm{Pl}}^{2}$ while $\left\langle X^{2}\right\rangle$ is about one order of magnitude smaller, see Fig. 2. The resonance stage ends when $\phi_{0}^{2}\left(t_{r}\right) \sim 5 \times 10^{-6} M_{\mathrm{Pl}}^{2} \gg\left\langle\phi^{2}\right\rangle$. If we again restrict ourselves to the interaction of the form $c \lambda \delta \Phi \phi^{4} /\left(4 M_{\mathrm{Pl}}\right)$, moduli are produced through the scatterings of the inflaton quanta and - at the end of the resonance stage $-\left\langle\Phi^{2}\right\rangle \sim 10^{-13} M_{\mathrm{Pl}}^{2}$.

The analytic estimate in eq. (7) well reproduces this numerical result for $\left\langle\Phi^{2}\right\rangle$. The corresponding ratio $\frac{n_{\Phi}}{s}$, derived from eq. (10), violates the bound (1) by seven orders of magnitude for $c=1$.

Another option is to have a coupling of the form $g^{2} \delta \Phi \phi^{2} X^{2} /\left(2 M_{\mathrm{Pl}}\right)$. In such a case the production of moduli cannot take place through oscillations at the initial stage of the parametric resonance, but only via inverse decays of already created fluctuations of the $X$ field. This is equivalent to say that the effect in the Green function method is not linear in the fluctuations, but at most quadratic. However, moduli are produced at the initial stage of the semiclassical thermalization and the result is not much different from the previous case, see Fig. 通.

The main difference during the evolution arises because the effective couplings in 
the system depend upon the avereage value of the fluctuations of the moduli field. This changes effectively the parameter $q$ and the time development of the resonance which is very sensitive to any change of $q$.

We can now use the Green-function method to give an estimate which approximately reproduces the results found in Fig. 3. The Fourier modes of the moduli fields at time $t$ can be written as

$$
\Phi_{\mathbf{k}}(t)=\frac{c g^{2}}{2 M_{\mathrm{Pl}}} \int_{0}^{t} d t^{\prime} \frac{\sin \left[\omega_{k}\left(t-t^{\prime}\right)\right]}{\omega_{k}} \phi_{0}^{2}\left(t^{\prime}\right) \int d^{3} p X_{\mathbf{p}}\left(t^{\prime}\right) X_{\mathbf{k}-\mathbf{p}}\left(t^{\prime}\right) .
$$

As expected, the moduli Fourier modes are quadratic in the fluctuations of the $X$ field. We can perform the time integral by assuming that the leading time dependence comes from the exponential growth of $X_{\mathbf{p}}$, which is proportional to $e^{\mu_{X} t}$, with $\mu_{X} \simeq$ $0.1 \sqrt{\lambda} \phi_{0}(0)$. Since the momentum distribution of the $X$ fluctuations is sharply peaked, the integral over $\mathbf{k}$ of the square of eq. (11) at the end of the resonance gives

$$
\left\langle\Phi^{2}\right\rangle \simeq\left[\frac{g^{2} \phi_{0}^{2}\left(t_{r}\right)\left\langle X^{2}\right\rangle}{4 M_{\mathrm{Pl}} \mu_{X} \omega_{k}\left(t_{r}\right)}\right]^{2} \simeq 10^{3} q^{2} \frac{\phi_{0}^{2}\left(t_{r}\right)\left\langle X^{2}\right\rangle^{2}}{M_{\mathrm{Pl}}^{4}} .
$$

Here we have used $\omega_{k} \sim \sqrt{\lambda} \phi_{0}$ as the typical frequency. From Fig. 3 we gather that, at the maximum, $\left\langle X^{2}\right\rangle \simeq 10^{-7} M_{\mathrm{Pl}}^{2}$, corrisponding to $\phi_{0}^{2}\left(t_{r}\right) \simeq 10^{-5} M_{\mathrm{Pl}}^{2}$. For $q=25$, eq. (12) gives $\left\langle\Phi^{2}\right\rangle \simeq 10^{-13} M_{\mathrm{Pl}}^{2}$, in fair agreement with our numerical results.

In all cases we also have calculated the particle occupation numbers to make sure that the lattice spacing and the integration box are suitably chosen and the relevant particle momenta are well represented. Particle number densities in the comoving reference frame are shown in Fig. 1 for the same model which is represented in Fig. 3 and at the time $\tau=180$. Let us also note that the production of moduli during the preheating stage should be analyzed by letting the moduli be coupled to the matter fields in all the interaction terms. Therefore, our estimates provide a lower limit on the abundance of moduli states.

Reassured by the capability of reproducing the numerical results by analytical means, we have also considered the quadratic inflaton model with quartic coupling to another field $X, V=\frac{1}{2} m^{2} \phi^{2}+\frac{1}{2} g^{2} \phi^{2} X^{2}$. The $q$-parameter is $q=g^{2} \phi_{0}^{2}(0) / 4 m^{2}$. It is also useful to introduce the redshifted resonance parameter at the end of the resonance stage $q_{r}=q\left(\phi_{0}\left(t_{r}\right) / \phi_{0}(0)\right)^{2}$. Parametric resonance can fully develop in an expanding Universe if $q_{r} \gtrsim 1$ [15]. Resonant production is most effective for fluctuations of fields which couple not too weakly but also not too strongly, those with $q_{r} \sim 1$. For $q_{r} \gg 1$, the maximal size of $X$ fluctuations is suppressed by non-linear effects [15, 16]. The zero mode rapidly drops at the end of the chaotization stage and all its energy is transferred 


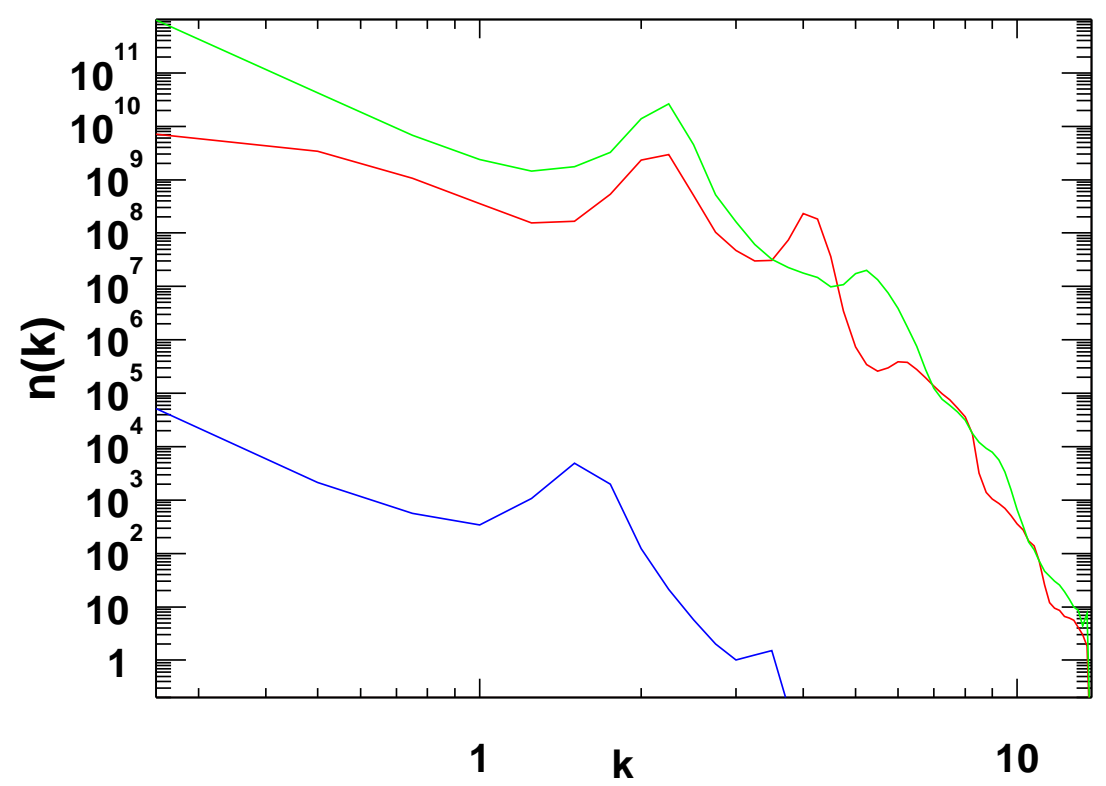

Figure 4: Particle number densities of $X$, inflaton and moduli fields, from top to bottom, at $\tau=180$ in the comoving reference frame, in units of $\sqrt{\lambda} \phi_{0}(0)$, for the same model studied in Fig. 3 .

to fluctuations [16]; the variance of the inflaton field is much larger than the value of the zero mode at the chaotization phase.

We now want to perform an analytical estimate of the number density of moduli fields for moderate $q_{r}$. If the mass term has a dependence on the modulus field $\Phi$, $m^{2}=m^{2}\left(\Phi / M_{\mathrm{Pl}}\right)$, this will introduce a trilinear coupling $m^{2} \phi^{2} \delta \Phi /\left(2 M_{\mathrm{Pl}}\right)$ between the quanta of the modulus and the inflaton quanta. In such a case, the Green-function method previously illustrated gives a modulus variance at the end of resonance equal to

$$
\left\langle\Phi^{2}\right\rangle \simeq \frac{m^{4}\left\langle\phi^{2}\right\rangle^{2}}{16 \omega^{2}\left(t_{r}\right) \mu^{2} M_{\mathrm{Pl}}^{2}} \simeq \frac{\left\langle\phi^{2}\right\rangle^{2}}{M_{\mathrm{Pl}}^{2}} .
$$

Here we have taken $\omega \sim m$ as the typical energy and $\mu \sim 0.2 m$ [13]. For $q_{r} \sim 1$, which corresponds to $q \sim 10^{4}$ [15], the inflaton completely decays into fluctuations during the chaotization phase; at the end of it $\phi_{0}^{2} \sim\left\langle\phi^{2}\right\rangle \sim n_{\phi} / m \sim 10^{-6} M_{\mathrm{Pl}}^{2}$. Therefore, from eq. (13), we obtain $n_{\Phi} / n_{\phi} \sim 10^{-6}$.

Notice that we could obtain the same estimate using the Boltzmann equation for the number density $n_{\Phi}$ :

$$
\dot{n}_{\Phi}+3 H n_{\Phi} \sim \frac{m^{4}}{\omega^{4}} \frac{n_{\phi}^{2}}{M_{\mathrm{Pl}}^{2}} .
$$


Assuming again $\omega \sim m$ and knowing that $n_{\phi}$ grows exponentially with rate $2 \mu$, we get $n_{\Phi} / n_{\phi} \sim n_{\phi} /\left(\mu M_{\mathrm{Pl}}^{2}\right)$.

At the end of the reheating stage, the energy density stored in the inflaton is converted into a relativistic thermal bath with temperature $T_{r} \sim\left(m n_{\phi}\right)^{1 / 4}$. The number density of moduli per entropy density will then be

$$
\frac{n_{\Phi}}{s} \sim \frac{n_{\Phi}}{n_{\phi}} \frac{T_{r}}{m} \sim 10^{-6} \frac{T_{r}}{m} .
$$

Since the COBE normalization gives $m \sim 10^{13} \mathrm{GeV}$, the upper bound on the reheating temperature becomes

$$
T_{r} \lesssim 10^{7} \mathrm{GeV}
$$

If the coupling of the modulus is $g^{2} \delta \Phi \phi^{2} X^{2} /\left(2 M_{\mathrm{Pl}}\right)$, the Green function method gives

$$
\left\langle\Phi^{2}\right\rangle \simeq\left[\frac{g^{2} \phi_{0}^{2}\left(t_{r}\right)\left\langle X^{2}\right\rangle}{4 M_{\mathrm{Pl}} \mu_{X} \omega\left(t_{r}\right)}\right]^{2} \simeq\left[\frac{q_{r}\left\langle X^{2}\right\rangle}{0.2 M_{\mathrm{Pl}}}\right]^{2} .
$$

Here we have assumed $\omega \sim m$ and $\mu_{X} \sim 0.2 m$. Repeating the same argument used above, we find $n_{\Phi} / s \sim 10^{7} q_{r}^{2}\left\langle X^{2}\right\rangle^{2} T_{r} /\left(M_{\mathrm{Pl}}^{4} m\right)$. Since at the end of the resonance phase $q_{r} \sim 1$, we find that the bound (1) gives a limit on the reheating temperature of $10^{5} \mathrm{GeV}$ if - for instance - we use $q=10^{6}$ and the maximum value of $\left\langle X^{2}\right\rangle \sim 10^{-6} M_{\mathrm{Pl}}^{2}$ [16].

So far we have always considered moduli couplings arising from the scalar potential, but similar results are also obtained when the moduli couplings are derived from nonminimal kinetic terms. For instance, from an interaction of the form $\delta \Phi \dot{\phi}^{2} / M_{\mathrm{Pl}}$ we estimate

$$
\left\langle\Phi^{2}\right\rangle \sim \frac{\dot{\phi}_{0}^{2}\left\langle\dot{\phi}^{2}\right\rangle}{M_{\mathrm{Pl}}^{2} \omega^{4}} \sim \frac{\phi_{0}^{2}\left\langle\phi^{2}\right\rangle}{M_{\mathrm{Pl}}^{2}}
$$

In the case of chaotic inflation with quadratic potential, eq. (18) is equivalent to eq. (13), valid for moduli coupled to the inflaton mass term.

\section{Moduli Production at Preheating in Hybrid In- flation}

In this section we study the moduli production during the preheating phase following a period of hybrid inflation [18]. This scenario involves two scalar fields, the inflaton field $\phi$, and the symmetry breaking field $\sigma$, and different mass scales and couplings. During inflation, the inflaton field $\phi$ rolls down along a flat potential while the Higgs field $\sigma$ is stuck at the origin providing the vacuum energy density driving inflation. However, when $\phi$ gets smaller than a critical value $\phi_{c}$, both fields roll down very quickly towards their present minima, completing the inflationary phase. 


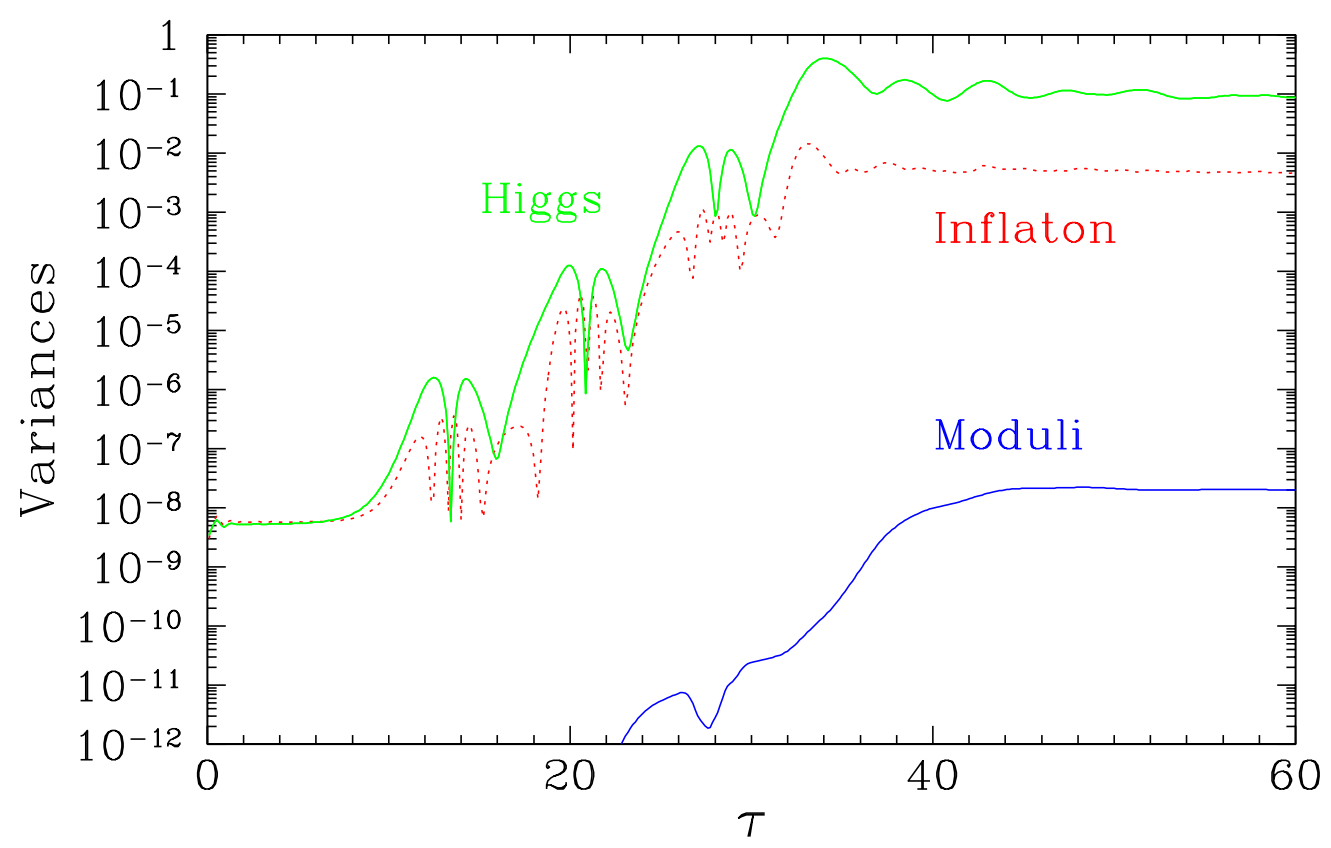

Figure 5: Variances of the inflaton, Higgs and moduli fields in units of $v^{2}$ in the hybrid model with $g^{2} / \lambda=10, \lambda=10^{-8}$ and $v=10^{-3} M_{\mathrm{Pl}}$. The moduli are coupled according to the $g^{2} \phi^{2} X^{2} \Phi /\left(2 M_{\mathrm{Pl}}\right)$ interaction.

We take the simplest hybrid inflation potential as suggested by Linde [18]

$$
V(\phi, \sigma)=\frac{\lambda}{4}\left(v^{2}-\sigma^{2}\right)^{2}+\frac{1}{2} m_{\phi}^{2} \phi^{2}+\frac{1}{2} g^{2} \phi^{2} \sigma^{2} .
$$

This potential has a valley of minima at $\sigma=0$ for $\phi^{2}>\phi_{c}^{2} \equiv \frac{\lambda}{g^{2}} v^{2}$. Most of the inflation occurs while $\phi$ is slowly rolling down from its initial value to $\phi_{c}$. During this phase $\sigma=0$ and the potential reduces to $V=\frac{\lambda}{4} v^{4}+\frac{1}{2} m_{\phi}^{2} \phi^{2}$. For $\phi<\phi_{c}$, the concavity of the potential in the $\sigma$ direction changes so that the $\phi$-axis is a ridge of unstable maxima.

Inflation ends because of the growth of quantum fluctuations in $\sigma$. For $\phi>\phi_{c}$, these are quickly damped out, but beneath $\phi_{c}$ they cause the fields to fall very quickly to global minima at $\phi=0$ and $\sigma^{2}=v^{2}$. There, they will execute damped oscillations.

The results of numerical integration for the case $v=10^{-3} M_{\mathrm{Pl}}$ are shown in Fig. 目, where we have supposed that the modulus is coupled to the inflaton field and the Higgs field via the coupling $g^{2} \Phi \phi^{2} \sigma^{2} /\left(2 M_{\mathrm{Pl}}\right)$. In hybrid models the coupling constant $\lambda$ does not have to obey the same restrictions as in the case of chaotic inflation. However, for the numerical integration we have chosen a rather low value, $\lambda=10^{-8}$. This is because

\footnotetext{
${ }^{3}$ For other hybrid inflation models, including those motivated by supersymmetry, see ref. [4].
} 
the particle number in the inflaton or Higgs excitations at the beginning of chaotization is $n(k) \sim 1 / \lambda$ and to be able to treat moduli fields classically, we need occupation numbers $n_{\Phi} \gg 1$. Since $n_{\Phi} \ll n(k)$, we need a rather small value of $\lambda$. For the run presented in Fig. 5 we have chosen $g^{2} / \lambda=10$.

The initial conditions were chosen in such a way that Higgs oscillations occur only around one of the minima, and domain creation and other effects leading to complete chaotisation during the first oscillation (see Ref. [19]) can be disregarded.

Our numerical findings show that the variance of the moduli fields does depend only upon the ratio $g^{2} / \lambda$.

The Green function method tells us that

$$
\left\langle\Phi^{2}\right\rangle \simeq \frac{g^{4}}{M_{\mathrm{Pl}}^{2}} \frac{\left\langle\phi^{2}\right\rangle^{2}}{\omega_{\phi}^{2}} \frac{\left\langle\sigma^{2}\right\rangle^{2}}{\omega_{\sigma}^{2}} \simeq \frac{g^{2}}{\lambda} \frac{\left\langle\phi^{2}\right\rangle^{2}}{M_{\mathrm{Pl}}^{2}},
$$

since the typical frequencies are given by $\omega_{\phi}^{2} \sim \lambda\langle\sigma\rangle^{2}$ and $\omega_{\sigma}^{2} \sim g^{2}\langle\sigma\rangle^{2}$. Using the same values of the couplings and mass scales adopted in Fig. 5 and reading from there the variance of the inflaton field $\left\langle\phi^{2}\right\rangle \sim 10^{-2} v^{2}$, we get $\left\langle\Phi^{2}\right\rangle \sim 10^{-9} v^{2}$, in fair agreement with our numerical results. Notice that the numerical values of the inflaton and Higgs variances at their maximum are well reproduced by $\left\langle\phi^{2}\right\rangle \sim\left(\lambda / g^{2}\right) v^{2}$ and $\left\langle\sigma^{2}\right\rangle \sim v^{2}$. Using these values we estimate $\left\langle\Phi^{2}\right\rangle \sim\left(\lambda / g^{2}\right)\left(v^{4} / M_{\mathrm{Pl}}^{2}\right)$, in good agreement with our numerical results and with the fact that these results depend only upon the ratio $g^{2} / \lambda$.

If the final reheating takes place via decay of the lightest particles in the system, i.e. the Higgs particles with mass squared $\lambda v^{2}$, the final moduli number density-to-entropyratio is given by

$$
\frac{n_{\Phi}}{s} \sim \frac{n_{\Phi}}{n_{\sigma}} \frac{T_{r}}{\sqrt{\lambda} v}
$$

which implies

$$
T_{r} \lesssim \sqrt{\lambda}\left(\frac{10^{-3} M_{\mathrm{Pl}}}{v}\right)\left(\frac{g^{2} / \lambda}{10}\right) 10^{11} \mathrm{GeV} .
$$

This result illustrates how, in certain ranges of parameters, the coherent production of moduli can become more dangerous than the generation of moduli via thermal scatterings.

\section{Modulino Production at Preheating}

So far we have been considering the generation of scalar moduli during the preheating stage. In this section we consider the production of the fermionic partners $\psi_{\Phi}$ of the moduli fields, sometimes dubbed modulini. As for the scalar moduli, the modulini are generically coupled to the matter fields through couplings suppressed by $M_{\mathrm{Pl}}$. 
Consider - for instance - the superpotential $W=\frac{\sqrt{\lambda}}{3} \phi^{3}$ giving rise to the inflaton potential $V=\lambda \phi^{4}$. If the coupling $\lambda$ is a function of $\Phi / M_{\mathrm{Pl}}$, where now $\Phi$ has to be considered as a superfield, the Lagrangian will contain the following coupling

$$
\frac{\sqrt{\lambda}}{M_{\mathrm{Pl}}} \bar{\psi}_{\Phi} \psi_{\phi} \phi^{2}
$$

where have denoted with $\psi_{\phi}$ the inflatino, the fermionic partner of the inflaton field.

We can again make use of the Green function method to obtain the Fourier component of $\psi_{\Phi}$. Using the retarded Green function for a fermionic field, we find

$$
\psi_{\Phi}(\mathbf{k}, t)=\frac{\sqrt{\lambda}}{M_{\mathrm{Pl}}} \int_{0}^{t} d t^{\prime}\left\{-i \gamma^{0} \sin \left[\omega_{k}\left(t-t^{\prime}\right)\right]-\frac{\vec{\gamma} \cdot \vec{k}}{\omega_{k}} \cos \left[\omega_{k}\left(t-t^{\prime}\right)\right]\right\} \phi_{0}^{2}\left(t^{\prime}\right) \psi_{\phi}\left(\mathbf{k}, t^{\prime}\right) .
$$

We can then approximatly estimate the quantity $\left\langle\bar{\psi}_{\Phi} \psi_{\Phi}\right\rangle$ as

$$
\left\langle\bar{\psi}_{\Phi} \psi_{\Phi}\right\rangle \simeq \frac{\lambda}{M_{\mathrm{Pl}}^{2}} \frac{\phi_{0}^{4}}{\omega^{2}}\left\langle\bar{\psi}_{\phi} \psi_{\phi}\right\rangle
$$

One can now use as a guide the recent results obtained in the theory of generation of Dirac fermions during and after inflation [20]. During the first inflaton oscillations, the Fermi distribution function of the inflatini is rapidly saturated up to some maximum value of the momentum $k$, i.e $n_{k} \simeq 1$ for $k \lesssim k_{\max }$ and it is zero otherwise. The resulting number density is therefore $n_{k} \sim k_{\max }^{3}$. The value of $k_{\max }$ is expected to be roughly of the order of the inverse of the time-scale needed for the change of the mass of the inflatini. In the model under consideration, such a mass changes by an amount $\sqrt{\lambda} \phi_{0}$ in a time scale $\left(\sqrt{\lambda} \phi_{0}\right)^{-1}$ and one expects $k_{\max } \sim \sqrt{\lambda} \phi_{0}$. Therefore

$$
\left\langle\bar{\psi}_{\phi} \psi_{\phi}\right\rangle \sim \lambda^{3 / 2} \phi_{0}^{3}(0)
$$

where we have used the fact that most of the fermion production takes place during the first oscillation. Under these circumstances, one can also assume that the number density of inflatini $n_{\psi_{\phi}}$ and modulini $n_{\psi_{\Phi}}$ is well measured by $\left\langle\bar{\psi}_{\phi} \psi_{\phi}\right\rangle$ and $\left\langle\bar{\psi}_{\Phi} \psi_{\Phi}\right\rangle$, respectively. This gives

$$
\frac{n_{\psi_{\Phi}}}{n_{\psi_{\phi}}} \sim \frac{\phi_{0}^{2}(0)}{M_{\mathrm{Pl}}^{2}}
$$

where we have taken $\omega \sim k_{\max }$ in Eq. (25).

Finally, the ratio of the number density of modulini in units of the entropy density $s \sim \rho^{3 / 4} \sim \lambda^{3 / 4} \phi_{0}^{3}$ is given by

$$
\frac{n_{\psi_{\Phi}}}{s} \sim\left(\frac{\lambda}{10^{-13}}\right)^{3 / 4}\left(\frac{\phi_{0}(0)}{0.3 M_{\mathrm{Pl}}^{2}}\right)^{2} 10^{-11}
$$


This result will contradict the bound (1) by about an order of magnitude when the energy density in the scalar field is transferred to the energy density of a hot gas of relativistic particles.

Similar considerations apply to the inflaton model with quadratic potential. Suppose there is a coupling of the form $m \phi^{2} \Phi / M_{\mathrm{Pl}}$ in the superpotential. This gives rise in the Lagrangian to a coupling of the modulini with the fermionic partners of the inflaton of the form $\frac{m}{M_{\mathrm{Pl}}} \bar{\psi}_{\Phi} \psi_{\phi} \phi$. Using the Green function method we again obtain $n_{\psi_{\Phi}} \sim n_{\psi_{\phi}}\left(\phi_{0}(0) / M_{\mathrm{Pl}}\right)^{2}$. This gives $n_{\psi_{\Phi}} / n_{\psi_{\phi}} \sim\left(\mathrm{m} / \mathrm{M}_{\mathrm{Pl}}\right)^{2} \sim 10^{-12}$ and the generation of dangerous relics is not large enough to provide a strong constraint on the reheating temperature.

In the case of the hybrid model though, the bound is more restrictive. In supersymmetric hybrid models, the coupling constants $\lambda$ and $g$ are related, and typically one finds $\lambda / g^{2} \sim 1$ [4]. The COBE normalization implies that typically $v \simeq 5 \times 10^{15} \mathrm{GeV}$. During the oscillations of the Higgs and the inflaton field, one expects that the number density of "Higgsini " $\psi_{\sigma}$ and inflatini $\psi_{\phi}$ is of the order of the number density of Higgs particles excited during the preheating phase, $n_{\psi_{\sigma}} \sim n_{\psi_{\phi}} \sim n_{\sigma} \sim \lambda^{3 / 2} v^{3}$. If the modulus couples to the Higgs superfield via the interaction $\sqrt{\lambda}\left(\Phi / M_{\mathrm{Pl}}\right) \sigma^{2} \phi$ in the superpotential, the number density of modulini is expected to be

$$
\frac{n_{\psi_{\Phi}}}{n_{\sigma}} \sim\left(\frac{v}{M_{\mathrm{Pl}}}\right)^{2}
$$

The corresponding bound on the reheating temperature can be obtained using the same arguments which led us to eq. (22); we find $T_{r} \lesssim \sqrt{\lambda}\left(5 \times 10^{15} \mathrm{GeV} / v\right) 10^{10} \mathrm{GeV}$.

\section{Conclusions}

In this paper we have investigated the production of scalar and fermionic moduli fields which occurs during the preheating stage after inflation. If produced with too large abundances, the late decays of these relics may jeopardize the successful predictions of standard big-bang nucleosynthesis. Since the moduli couple to any form of matter at least gravitationally, their generation is unescapable in the large class of inflationary models which have a preheating stage. They are generated as coherent states initially. From this point of view, the production mechanism of moduli differs from the generation mechanism of squeezed states during the parametric resonance which has been studied in recent years. We have shown that moduli are efficiently produced during the preheating stage. The corresponding upper bound on the reheating temperature is model dependent and sensitive to the details of the stage of thermalization following 
the period of parametric resonance. It is often tighter than the bound of $10^{9} \mathrm{GeV}$ found considering the generation of moduli via thermal scatterings during reheating.

\section{References}

[1] G. Coughlan, W. Fischler, E. Kolb, S. Raby, and G. Ross, Phys. Lett. B131, 59 (1983); J. Ellis, D. V. Nanopoulos, and M. Quirós, Phys. Lett. B174, 176 (1986);

B. de Carlos, J.A. Casas, F. Quevedo, and E. Roulet, Phys. Lett. B318, 447 (1993);

T. Banks, D. Kaplan, and A. Nelson, Phys. Rev. D49, 779 (1994).

[2] D. Lindley, Ap. J. 294, 1 (1985); J. Ellis et al., Nucl. Phys. 259, 175 (1985); S. Dimopoulos et al., Nucl. Phys. B311, 699 (1988); M.H. Reno and D. Seckel, Phys. Rev. D37, 3441 (1988); J. Ellis et al., Nucl. Phys. B373, 399 (1992); E. Holtman, M. Kawasaki, K. Kohri, and T. Moroi, Phys. Rev. D60, 023506 (1999).

[3] J. Ellis, A. Linde, and D. Nanopoulos, Phys. Lett. B118, 59 (1982); D. Nanopoulos, K. Olive, and M. Srednicki, Phys. Lett. B127, 30 (1983); J. Ellis, J. Kim, and D. Nanopoulos, Phys. Lett. B145, 181 (1984).

[4] D. H. Lyth and A. Riotto, Phys. Rept. 314, 1 (1999).

[5] M. Kawasaki and T. Moroi, Prog. Theor. Phys. 93, 879 (1995).

[6] A. S. Goncharov, A. D. Linde and M. I. Vysotsky, Phys. Lett. B147, 279 (1984).

[7] G. F. Giudice, I. Tkachev and A. Riotto, JHEP 9908, 009 (1999).

[8] G. Felder, L. Kofman and A. Linde, JHEP0002, 027 (2000).

[9] R. Kallosh, L. Kofman, A. Linde and A. Van Proeyen, Phys. Rev. D 61, 103503 (2000).

[10] G. F. Giudice, A. Riotto and I. Tkachev, JHEP 9911, 036 (1999).

[11] R. Kallosh, L. Kofman, A. Linde and A. Van Proeyen, Class. Quant. Grav. 17, 4269 (2000).

[12] H. P. Nilles, M. Peloso and L. Sorbo, hep-ph/0102264; H. P. Nilles, M. Peloso and L. Sorbo, to appear. 
[13] L. Kofman, A. Linde and A. A. Starobinsky, Phys. Rev. Lett. 73, 3195 (1994);

L. Kofman, A. Linde and A. A. Starobinsky, Phys. Rev. D 56, 3258 (1997) hepph/9704452].

[14] S. Y. Khlebnikov and I. I. Tkachev, Phys. Rev. Lett. 77, 219 (1996).

[15] S. Y. Khlebnikov and I. I. Tkachev, Phys. Lett. B390, 80 (1997).

[16] S. Y. Khlebnikov and I. I. Tkachev, Phys. Rev. Lett. 79, 1607 (1997).

[17] E. W. Kolb, A. Linde and A. Riotto, Phys. Rev. Lett. 77, 4290 (1996); E. W. Kolb, A. Riotto and I. I. Tkachev, Phys. Lett. B 423, 348 (1998).

[18] A. Linde, Phys. Rev. D 49, 748 (1994).

[19] G. Felder et al., hep-ph/0012142.

[20] J. Baacke, K. Heitmann, and C. Patzold, Phys. Rev. D58, 125013 (1998); P. B. Greene and L. Kofman, Phys. Lett. B448, 6 (1999); V.A. Kuzmin and I.I. Tkachev, Phys. Rev. D59, 123006 (1999); G.F. Giudice, M. Peloso, A. Riotto, and I. Tkachev, JHEP 9908014 (1999); D.J. Chung, E.W. Kolb, A. Riotto and I.I. Tkachev, Phys. Rev. D 62, 043508 (2000). 